\title{
Immune thrombocytopenia in two unrelated Fanconi anemia patients - a mere coincidence?
}

\section{OPEN ACCESS}

Edited by:

Jordan Orange,

Baylor College of Medicine, USA

Reviewed by:

Sarah Kogan Nicholas,

Baylor College of Medicine, USA

Nicholas L. Rider,

Texas Children's Hospital and Baylor

College of Medicine, USA

${ }^{*}$ Correspondence:

Angela Wawer,

Department of Pediatrics, Division of

Pediatric Hematology/Oncology,

Technische Universität München

(TUM), Kölner Platz 1 ,

Munich 80804, Germany angela.wawer@lrz.tu-muenchen.de;

Markus G. Seidel,

Department of Pediatrics and

Adolescent Medicine, Division of

Pediatric Hematology/Oncology,

Medical University Graz,

Auenbruggerplatz 38, Graz A-8036,

Austria

markus.seidel@medunigraz.at

${ }^{\dagger}$ Contributed equally and should be

considered aequo loco.

Specialty section:

This article was submitted to Pediatric Hematology and Immunology, a section of the journal Frontiers in

Pediatrics

Received: 19 April 2015

Accepted: 15 May 2015

Published: 08 June 2015

Citation:

Karastaneva A, Lanz S, Wawer A, Behrends U, Schindler D, Dietrich R, Burdach S, Urban C, Benesch M and

Seidel MG (2015) Immune thrombocytopenia in two unrelated

Fanconi anemia patients - a mere coincidence?

Front. Pediatr. 3:50. doi: 10.3389/fped.2015.00050

\author{
Anna Karastaneva ${ }^{1 \dagger}$, Sofia Lanz ${ }^{1 \dagger}$, Angela Wawer ${ }^{2 *}$, Uta Behrends ${ }^{2}$, Detlev Schindler ${ }^{3}$, \\ Ralf Dietrich ${ }^{4}$, Stefan Burdach ${ }^{2}$, Christian Urban ${ }^{1}$, Martin Benesch $^{1}$ and \\ Markus G. Seidel ${ }^{1 *}$

\begin{abstract}
'Division of Pediatric Hematology/Oncology, Department of Pediatrics and Adolescent Medicine, Medical University Graz, Graz, Austria, ${ }^{2}$ Division of Pediatric Hematology/Oncology, Department of Pediatrics, Technische Universität München, Munich, Germany, ${ }^{3}$ Institute of Human Genetics, Biozentrum, University of Würzburg, Würzburg, Germany, ${ }^{4}$ German Fanconi Anemia Support Group, Unna, Germany
\end{abstract}

Thrombocytopenia and pancytopenia, occurring in patients with Fanconi anemia (FA), are interpreted either as progression to bone marrow failure or as developing myelodysplasia. On the other hand, immune thrombocytopenia (ITP) represents an acquired and often self-limiting benign hematologic disorder, associated with peripheral, immune-mediated, platelet destruction requiring different management modalities than those used in congenital bone marrow failure syndromes, including FA. Here, we describe the clinical course of two independent FA patients with atypical - namely immune - thrombocytopenia. While in one patient belonging to complementation group FA-A, the ITP started at 17 months of age and showed a chronically persisting course with severe purpura, responding well to intravenous immunoglobulins (IVIG) and later also danazol, a synthetic androgen, the other patient (of complementation group FA-D2) had a self-limiting course that resolved after one administration of IVIG. No cytogenetic aberrations or bone marrow abnormalities other than FA-typical mild dysplasia were detected. Our data show that acute and chronic ITP may occur in FA patients and impose individual diagnostic and therapeutic challenges in this rare congenital bone marrow failure/tumor predisposition syndrome. The management and a potential context of immune pathogenesis with the underlying marrow disorder are discussed.

Keywords: immune thrombocytopenia, Fanconi anemia, bone marrow failure syndrome, DNA repair defect, Evans syndrome, danazol, FANCA, FANCD2

\section{Introduction}

Thrombocytopenia and pancytopenia are frequent hematologic manifestations of Fanconi anemia (FA) ascribed to a varying, increasing degree of congenital bone marrow failure and developing myelodysplasia. Treatment options range from none (observation) to therapeutic administration of androgens and, in case of transfusion dependence or signs of (pre-) malignancy, e.g., cytogenetic aberrations indicative of clonal evolution, hematopoietic stem cell transplantation. At least 17 genes are known to be involved in the pathogenesis of FA. Stringent genotype-phenotype correlations are rare in classical FA and have consistently only been observed for variant groups, e.g., FA-D1 and FA-N (1-3). 
Immune thrombocytopenia (ITP) is observed as an acquired acute and self-limited benign post- or para-infectious or idiopathic cytopenia in otherwise healthy children that, if treatment is required, usually responds well to high-dose intravenous immunoglobulins (IVIG) and/or corticosteroids (4). Chronic ITP may develop and indicate an underlying immune hematologic disorder (5). Refractory chronic ITP with bleeding diathesis sometimes requires additional immunosuppressive or thrombopoietin agonist treatment, or splenectomy. We observed the clinical course in two patients with thrombocytopenia of immune origin in the context of FA. Here, we delineate the diagnostic and therapeutic challenges of this previously unnoticed concurrence and discuss potential implications.

\section{Patients and Methods}

Two patients who have been diagnosed, treated, and prospectively monitored for FA at pediatric hematology/oncology departments at the Medical University Graz and the Technische Universität München (TUM) were found to suffer from ITP in 2014. The data presented here were obtained by retrospective chart review. Laboratory tests were performed according to clinical needs and routine standard procedures. After mitomycin C-mediated induction of G2 arrest and chromosomal breaks and/or complementation group analysis for FA, genetic testing was performed by means of multiplex ligation-dependent probe amplification (MLPA) and exon-scanning sequencing of genomic DNA of all exons of FANCA (patient 1) and of FANCD2 (patient 2). Both patients are registered within the German registry for FA FAR01 of the German Society for Paediatric Oncology and Haematology $(\mathrm{GPOH})$. The present study was performed upon informed consent in accordance with Declaration of Helsinki and approval of the responsible internal review boards.

\section{Results}

\section{Patient Report 1}

The first patient is a currently 3-year-old female, who was termborn and small for gestational age. Additionally to intrauterine growth retardation, other stigmata consistent with FA were present (Table 1). Diagnosis was confirmed in vitro by typical diepoxybutane- and mitomycin c-induced double strand break induction, G2 arrest, and a FANCA mutation (EX2_6del heterozygous, second mutation yet elusive). Esophageal atresia type IIIB required repetitive dilatation, until surgical intervention (Nissen fundoplication) at 17 months of age was undertaken. Prior to surgery, peripheral blood counts were stable within normal ranges. Baseline bone marrow evaluation had not been performed. Postoperatively, an isolated mild thrombocytopenia (minimum 70,000/ $\mu \mathrm{l}$ ) was observed. Platelet counts recovered spontaneously to near normal ranges $(>100,000 / \mu \mathrm{l})$ within the next months. A second abrupt and more pronounced platelet decline $(23,000 / \mu \mathrm{l})$ along with generalized petechial exanthema occurred 4 months later following anesthesia for an esophageal passage imaging study. Response to platelet transfusions was only transitory (Figure 1A), and repeated platelet transfusions were given to control the purpura. However, a brief increase of platelet numbers was always followed by a rapid decline (Figure 1A). An evaluation for allogeneic stem cell transplantation and a donor search were initiated.

The early manifestation of thrombocytopenia in FA, refractory to administration of platelet concentrates, warranted a more in-depth hematological evaluation. Apart from reduced platelet numbers, peripheral blood counts and erythrocyte indices remained normal during the period of observation (Table 1). Surprisingly, bone marrow examination did not show bone marrow failure or myelodysplastic syndrome (MDS; Table 1). In contrast, it revealed a marrow of normal cellularity, a megakaryocyte count in the upper normal range, and only mild dysplasia of all three cell lines, considered "normal for FA". Neither signs of malignant infiltration and transformation to MDS were found, nor were any clonal chromosomal aberrations such as $1 \mathrm{q}+, 3 \mathrm{q}+, 7 / 7 \mathrm{q}-, 5 / 5 \mathrm{q}-$, or trisomy 8 detectable. In the absence of a clinically apparent infection, PCR testing for HHV6, Parvovirus B19, CMV, and EBV from the bone marrow aspirate were performed with negative results. Assuming an immune-mediated mechanism IVIG were given, leading to an increase of platelets. Subsequently, the thrombocytopenia demonstrated a chronically persisting course with severe purpura, responding well to IVIG (Figure 1A). Anti-CMV IgG (analyzed before IVIG administration) and IgM were positive in blood sample, as was CMV nucleic acid in the urine $\left(4-6 \times 10^{4}\right.$ copies/mL, Table 1), suggesting the presence of or recent recovery from a CMV infection, which represents a potential trigger of immune-mediated platelet destruction. The patient received a total of three platelet transfusions and seven IVIG infusions (5 with $0.8 \mathrm{~g} / \mathrm{kg}$ body weight and two infusions with $0.5 \mathrm{~g} / \mathrm{kg}$ body weight) within the first 6 months after presentation with ITP, but signs of bleeding (dry and wet purpura) and recurring thrombocytopenia persisted (Figure 1A). A short attempt of corticosteroid treatment with dexamethasone led to moderate response (platelet increase from 24,000 to $91,000 / \mu \mathrm{l}$ ) but was terminated by the parents after 4 days because of inacceptable temper changes of the girl. Treatment with danazol, a synthetic androgen, was initiated at $5 \mathrm{mg} / \mathrm{kg} /$ day 5 months after the onset of thrombocytopenia, which slowly led to a satisfactory response with regards to bleeding tendency and was accompanied by a platelet count stabilization between 40,000 and $140,000 / \mu$ l. Thus, the need of interventional IVIG treatment was substantially reduced $(n=2$ per 6 months versus 7 per previous 6 months; Figure 1A). Side effects of danazol were weight gain (change of percentile from 10th to 25 th within 9 months) and mood swings, both considered unspecific and tolerable for the patient by her family and treating physicians. Virilization or other endocrine abnormalities were not observed during the first 9 months of danazol treatment. The androgen dose could be reduced to $2 \mathrm{mg} / \mathrm{kg}$ after 6 months without change of platelet counts and will be further reduced if platelet counts remain stable. A recent follow-up bone marrow aspiration and trephine biopsy 1-year after the initial manifestation of thrombocytopenia showed no dynamics as compared to the previous analyses (not shown).

\section{Patient Report 2}

The second patient, a 7-year-old female was born on term with intrauterine growth retardation and multiple congenital abnormalities typical for FA. Furthermore, she failed to thrive (Table 1). 
TABLE 1| Patient characteristics of two girls with FA and ITP.

\section{Patient 1}

3 years, female

\section{Genotype}

FANCA compound heterozygous: EX2_6del; 2nd yet elusive

Clinical presentation at birth and at diagnosis of FA

\section{Pregnancy [week] \\ Birth weight [g]}

Birth length [cm]

Head circumference [cm]

Upper limb

Thumb hypoplasia

Thumb aplasia

Lower limb

Congenital hip dysplasia

Head and face

Microcephaly

Microphthalmia

Growth

Small stature

Gl system

Esophageal atresia

Cardiac system

Congenital heart defect

Other

Impaired hearing

Hypogammaglobulinemia

Blood type

Bone marrow at diagnosis of ITP

\section{$38+3$ \\ 2015 (<3rd\%) \\ $44(<3 \mathrm{rd} \%)$ \\ $30.5(<3 r d \%)$}

Right IIla-b; left II

$-$

$-$

$-3 S D^{e}$

$+$

$-2 S D$

IIIb

$-$

$+$

+ (transiently)

0, Rh: positive

Normocellular, discreet dysplasia and atypia of all compartments; megakaryopoiesis numerically in the upper normal range, $10 \%$ of megakaryocytes mono-hypolobulated, no blasts

\section{Patient 2}

7 years, female
FANCD2 compound heterozygous: 3-bp-deletion; missense substitution at codon 815

$38+2$

$2280(<10$ th $\%)$

$46(<10$ th $\%)$

32 (10-25th\%)

Right

Left

$+$

$-3 S D$

$+$

$-4 S D$

$\operatorname{VSD}^{f}$

0, Rh: positive

Moderately hypocellular, Blasts beneath 1\%, megakaryocytes without dysplasia, but clearly reduced and with hyper-segmented nuclei suspicious of MDS transformation

\section{Patient 1}

\section{Complete blood counts \\ (selected parameters)}

Hemoglobin [ $\mathrm{g} / \mathrm{dll}]$

Reticulocytes [T/]

Mean corpuscular volume [fl]

White blood cells $[/ \mu \mathrm{l}]$

Lymphocytes $[/ \mu \mathrm{l}]$

Neutrophil granulocytes $[/ \mu l]$

Cellular immune system

CD3 + T cells $[/ \mu l]$

CD3 + CD $4+$ T cells $[/ \mu l]$

$\mathrm{CD} 3+\mathrm{CD} 8+\mathrm{T}$ cells $[/ \mu \mathrm{l}]$

CD3-CD56 + NK cells $[/ \mu l]$

TREC s copies per $10^{e}$ CD3 + CD45+

CD19+ B cells $[/ \mu l]$

$\mathrm{TCRa} / \mathrm{b}+\mathrm{CD} 4-\mathrm{CD} 8-\mathrm{CD} 3+$

$\mathrm{CD} 19+\mathrm{CD} 27+\operatorname{lgD}+$

$\mathrm{CD} 19+\mathrm{CD} 27+\operatorname{lgD}-$

\section{Median (Min; Max)}

$12.4^{\mathrm{j}}(9.90 ; 14.8)$

$0.081^{\mathrm{j}}(0.042 ; 0.145)$

$81.2^{\mathrm{j}}(76.8 ; 93.2)$

$6640^{j}(3850 ; 12400)$

$3800^{j}(1700 ; 7300)$

$2210^{j}(670 ; 8800)$

3555 | normal $^{j}$

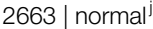

635 | normal $^{j}$

85 | moderately reduced

60500 | normal $^{j}$

617 | normal $^{j}$

$<2 \%$ of $T$ cells | normal

$>2 \%$ of $B$ cells | normal

$>2 \%$ of $B$ cells | normal

\section{Patient 2}

\section{Median (Min; Max)}

$12.5^{\mathrm{j}}(11.4 ; 13.7)$

$0.04^{\mathrm{j}}(0.02 ; 0.09)$

$86.8^{\mathrm{j}}(84 ; 91.8)$

$4600^{j}(2800 ; 7200)$

$4080^{\mathrm{j}}(2260 ; 7310)$

1500 (1050; 3740)

n.d.

n.d.

n.d.

n.d.

n.d.

n.d.

n.d.

n.d.

n.d.

Patient 1

Patient 2

$61 / 12$

Age: $9 / 12$

$18 / 12$

1038 | normal

n.a. ${ }^{a}$ 


\begin{tabular}{|c|c|c|c|}
\hline & \multicolumn{2}{|l|}{ Patient 1} & \multirow{2}{*}{$\begin{array}{l}\text { Patient } 2 \\
61 / 12\end{array}$} \\
\hline & Age: $9 / 12$ & $18 / 12$ & \\
\hline $\operatorname{lgG} 2[\mathrm{mg} / \mathrm{dL}]$ & $34.41 \mid$ low $^{\mathrm{g}}$ & $69.5 \mid$ normal $^{\mathrm{h}}$ & n.a. \\
\hline IgG3 [mg/dL] & 24.58 |normal $^{9}$ & $\left.73.83\right|_{\text {normal }} ^{\text {h }}$ & n.a. \\
\hline lgG4 [mg/dL] & $0.66 \mid$ normal $^{9}$ & $\left.0.39\right|_{\text {normal }^{\mathrm{h}}}$ & n.a. \\
\hline $\lg A[\mathrm{mg} / \mathrm{dL}]$ & 27.5 |normal $^{9}$ & $\left.54.3\right|_{\text {normal }^{h}}$ & $\left.108\right|_{\text {normal }} ^{i}$ \\
\hline $\operatorname{lgM}[\mathrm{mg} / \mathrm{dL}]$ & 50.9 |normal $^{g}$ & $90.6 \mid$ normal $^{\text {h }}$ & 44 | normali \\
\hline $\lg E[\mathrm{IU} / \mathrm{L}]$ & 4.2 | normal $^{9}$ & n.d. ${ }^{b}$ & n.a. \\
\hline Anti-diphtheria toxoid (DT) antibodies (Ab) [IU/L] & 2.47 | normal $^{g, c}$ & $\left.2.37\right|_{\text {normalh,c }} ^{\text {h,c }}$ & n.d. \\
\hline Anti-tetanus toxoid (TT) Ab [IU/L] & $\left.4.15\right|_{\text {normal }} ^{g, c}$ & $2.48 \mid$ normal $^{\mathrm{h}, \mathrm{c}}$ & n.d. \\
\hline Anti-pneumococcus polysaccharide (PCP) Ab [mg/L] & 42.96 |normal $^{\mathrm{g}, \mathrm{c}}$ & $\left.117.31\right|_{\text {normal }^{\mathrm{h}, \mathrm{c}}}$ & n.d. \\
\hline Anti-Haemophilus influenza B polysaccharide (HIB) Ab [mg/L] & $3.16 \mid$ normal $^{\mathrm{g}, \mathrm{c}}$ & $5.1 \mid$ normal $^{h, c}$ & n.d. \\
\hline \multicolumn{4}{|l|}{ Autoantibodies at diagnosis of ITP } \\
\hline Coombs test direct & \multicolumn{2}{|c|}{ Negative } & Negative \\
\hline Anti-platelet antibodies & \multicolumn{2}{|c|}{ Negative } & $\begin{array}{l}\text { Positive: } \\
\text { Gpllb/IIla }\end{array}$ \\
\hline \multirow[t]{2}{*}{ ANA } & \multicolumn{2}{|c|}{ Negative } & n.d. \\
\hline & \multicolumn{2}{|l|}{ Patient 1} & Patient 2 \\
\hline \multicolumn{4}{|l|}{ Microbiological results at diagnosis of ITP } \\
\hline Anti-Parvo B19 IgM, IgG & \multicolumn{2}{|l|}{ Negative } & Negative \\
\hline Anti-CMV IgM & \multicolumn{2}{|l|}{ Positive } & Negative \\
\hline Anti-CMV IgG & \multicolumn{2}{|l|}{ Positive } & Negative \\
\hline Anti-EBV lgM, lgG & \multicolumn{2}{|l|}{ Negative } & Negative \\
\hline Anti-HHV6 IgM & \multicolumn{2}{|l|}{ Negative } & Negative \\
\hline Anti-HHV6 IgG & \multicolumn{2}{|l|}{ Negative } & Positive \\
\hline HIV antigen and antibody & \multicolumn{2}{|l|}{ Negative } & Negative \\
\hline Anti-HCV & \multicolumn{2}{|l|}{ Negative } & Negative \\
\hline Anti-HBs lgG & \multicolumn{2}{|l|}{ Negative $^{\mathrm{C}}$} & Negative \\
\hline Anti-HBc lgG & \multicolumn{2}{|l|}{ Negative } & Negative \\
\hline Anti-VZV IgM & \multicolumn{2}{|l|}{ n.d. } & Negative \\
\hline Anti-VZV IgG & \multicolumn{2}{|l|}{ Negative } & Positive \\
\hline Anti-measles lgG & \multicolumn{2}{|l|}{ Positive $^{c}$} & Positive $^{c}$ \\
\hline \multicolumn{4}{|l|}{ Stool } \\
\hline Rotavirus AG ELISA & \multicolumn{2}{|l|}{ n.d. } & Positive \\
\hline Norovirus AG ELISA & \multicolumn{2}{|l|}{ n.d. } & Negative \\
\hline Adenovirs AG ELISA & \multicolumn{2}{|l|}{ n.d. } & Negative \\
\hline \multicolumn{4}{|l|}{ Urine } \\
\hline CMV nucleic acid & $\begin{array}{l}\mathbf{4 - 6} \times \mathbf{1 0}^{\mathbf{4}} \\
\text { copies } / \mathrm{mL}^{\prime}\end{array}$ & & n.d. \\
\hline Blood plasma nucleic acid detection & & & \\
\hline PCR for CMV; EBV; AdV; HSV1,2; HHV6, ParvoB19; VZV; Enterovirus & Negative & & n.d. \\
\hline
\end{tabular}

${ }^{a}$ n.a., data not available or not done before IVIG administration.

${ }^{b}$ n.d., not done.

${ }^{c}$ After vaccination.

e $S D$, standard deviation.

${ }^{f} V S D$, ventricular septal defect I.

${ }^{9-i}$ Age-adjusted reference values.

glgG: 223-1099; IgG1: 140-620; IgG2: 41-130; IgG3: 11-85; IgG4: 0-0,8; IgA: 1-73; IgM: 8-100; DT IgG: >1; TT IgG: 0,02-3,12; PCP IgG: 0,9-93; HIB IgG: 0,08-9,2.

hlgG: 344-1180; IgG1: 220-720; IgG2: 50-180; IgG3:14-91; IgG4: 0-40,8; IgA: 2-98; IgM: 12-104; DT IgG: >1; TT IgG: 0,04-3,92; PCP IgG: 0,9-29,2; HIB IgG: 0,16-40,8.

ilgG: 411-1435; IgA: 34-214; IgM: 15-115.

iNormal according to age-dependent reference ranges.

${ }^{k}$ As described in the medical reports, precise data missing.

'At three occasions within 9 months.

Laboratory parameters in "bold" indicate pathologic results.

A compound heterozygous FANCD2 mutation confirmed the diagnosis of FA (compound heterozygous for a 3-bp-deletion and a missense substitution at codon 815) (6). Suddenly, at the age of 6 years severe thrombocytopenia $(13,000 / \mu l)$ was observed incidentally during a hospitalization for rotavirus gastroenteritis, while the red and white blood counts remained stable. Other (e.g., myelosuppressive) viral infections were ruled out serologically (Table 1). Similarly to the first patient, bone marrow aspiration and a trephine biopsy were performed immediately to verify suspected bone marrow failure, but no abnormalities other than FA-typical mild dysplasia, nor any cytogenetic aberrations were detected (Table 1). In the absence of myelosuppression or marrow failure, further evaluation was directed toward immune pathogenesis of the low platelet count. Positive antiplatelet antibodies against GpIIb/IIIa corroborated the diagnosis of ITP. Thrombocytopenia resolved after a single infusion of IVIG (Figure 1B), and 


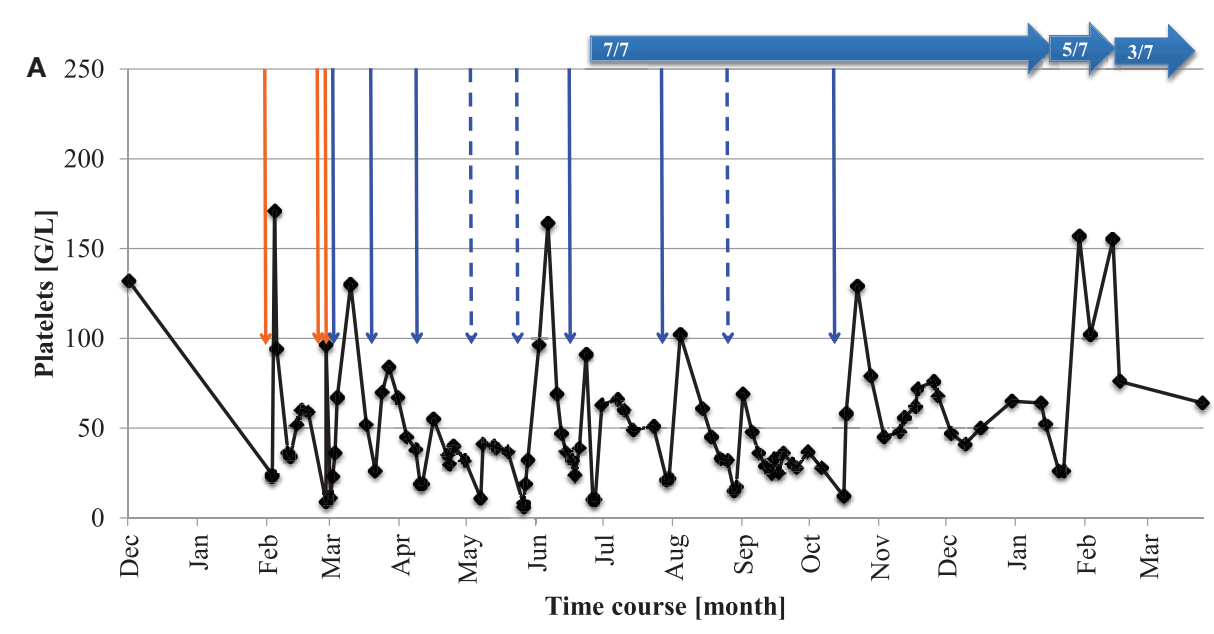

Danazol 50mg, given on days per week $(7 / 7 ; 5 / 7 ; 3 / 7)$

Platelet Concentrate $\quad \Rightarrow$ IVIG $0.8 \mathrm{~g} / \mathrm{kg}$ body weight $\quad \rightarrow \quad$ IVIG $0.5 \mathrm{~g} / \mathrm{kg}$

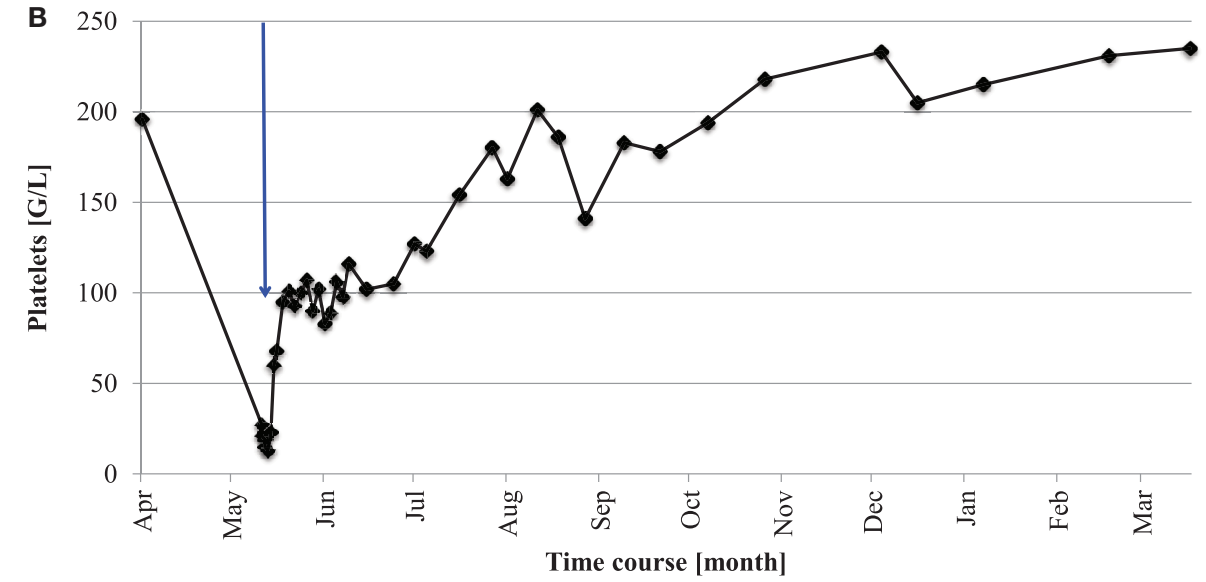

$\Rightarrow$ IVIG $0.8 \mathrm{~g} / \mathrm{kg}$ body weight

FIGURE 1 | Platelet count and treatment of two girls with ITP and FA over time.

platelet counts remained stable thereafter during 1 year after the initial presentation of ITP.

\section{Discussion}

The simultaneous occurrence of two diagnostic entities, that have the potential to affect the platelet count through different, apparently even reciprocal pathogenetic mechanisms, namely ITP and FA, raised both diagnostic and therapeutic challenges. While in one patient with a FANCA mutation, the ITP started at 17 months of age and showed a chronically persisting course with severe purpura, that responded to IVIG and synthetic androgen therapy, the other patient (with FANCD2 mutations) had a self-limited course that resolved after a single administration of IVIG. Other than FA-typical mild dysplasia, neither bone marrow abnormalities nor cytogenetic aberrations were detected. An association between platelet decline and anesthesia cannot be excluded in patient 1 , because thrombopenia initially manifested shortly after gastroduodenoscopy in anesthesia. A complete blood count had not been analyzed immediately before general anesthesia.

Three possible interpretations for the concurrence of ITP and FA may be discussed, which are pathogenetically relevant and have implications for decisions of further treatment options. First, the unusual combination of FA and ITP might be interpreted as coincidental - presuming that ITP developed independently from the underlying FA. ITP occurs in 5 of 100,000 children per year, and FA is the most common inherited bone marrow failure syndrome (IBMFS), frequently underdiagnosed in patients without phenotypic abnormalities (7, 8). Additionally, ITP may be associated with CMV infection as present in the first patient $(9,10)$. Following this hypothesis, the therapeutic approach to thrombocytopenia is supposed to adhere to the general recommendations for treatment of ITP. Accordingly, both reported patients demonstrate satisfactory response to IVIG, known as first line therapeutic option for ITP (4). 
Although its effectiveness is largely attributed to immunomodulating activity and considered an indirect evidence for an immune pathogenesis of an acute de novo thrombocytopenia, the exact mechanism of action of IVIG in ITP is still not entirely understood $(11,12)$. Interestingly, one study suggests a possible enhancement of thrombopoiesis by induction of endogenous thrombopoietin production following IVIG administration (12). Theoretically, in this case, platelet turnover might be positively influenced by IVIG both in ITP and FA. Currently, data for the efficacy of IVIG application in patients with FA are lacking. We did not treat patient 1 with thrombopoietin receptor agonists, because we generally aim to avoid hematopoietic growth factor administration in tumor predisposition syndromes. Patients with FA usually respond to treatment with androgens with an increase of erythropoiesis and, to a lesser extent, also thrombopoiesis (13-15). Likewise, a therapeutic efficacy of danazol is known in chronic ITP and other autoimmune hematologic disorders (Evans syndrome, autoimmune hemolytic anemia) (15-18). The exact mechanism of action of synthetic androgens is unknown, but immunomodulation is suspected to be involved (15).

Second, another explanation for the reported events could be that the underlying stem cell impairment facilitated or induced a subsequent immunologic reaction similar to that seen in refractory cytopenia of childhood and severe aplastic anemia (RCC/SAA) and thus, ITP might be considered "a feature of FA." In this case, bone marrow transplantation would have to be considered an obvious therapeutic strategy like in MDS in FA (19). Thrombocytopenia is a well-known first manifestation of beginning bone marrow failure in FA (19), often associated with macrocytosis and progessive myelodysplasia. However, macrocytosis was absent as was myelosuppression in bone marrow analyses of both patients revealing normal cellularity, and even megakaryocytosis in the first patient. Additionally, none of the detected FA-associated gene mutations in our patients (FANCA and FANCD2 mutations) are typically linked to early transformation to MDS or malignancy such as, e.g., FANC IVS5 in Ashkenazi Jews, FANCD1/BRCA2, or FANCN mutations (20-24). Furthermore, patient 2 recovered soon after a single dose of IVIG, and patient 1 repeatedly showed normalization of platelet numbers upon IVIG, indicating a healthy regenerative capacity. Thus, MDS or bone marrow failure was unlikely to be the cause of thrombocytopenia seen in the presented FA patients aged 21 months and 6 years.

Third, a more complex, multifactorial etiopathogenensis of thrombocytopenia might be suspected in FA patients. A toxic effect of preceding anesthesia in patient 1 and detected CMV in patient 1 and Rotavirus in patient 2 or another undetected viral infection triggering immunologic events might have more pronounced effects in the setting of a "weak" bone marrow predisposed toward dysplasia than in healthy bone marrow. While MDS is usually unresponsive to IVIG, danazol is frequently used

\section{References}

1. Schneider M, Chandler K, Tischkowitz M, Meyer S. Fanconi anaemia: genetics, molecular biology, and cancer - implications for clinical management in children and adults. Clin Genet (2014). doi:10.1111/cge.12517 to treat cytopenia in FA and Dyskeratosis congenita $(19,25)$. Indirect evidence of immunoglobulin-supported remission of thrombocytopenia in patient 2 and the good response of platelet counts to IVIG (and later danazol) in patient 1, together with the only mild, benign bone marrow abnormalities described, support the hypothesis of an immune-mediated pathogenesis, but other contributing factors facilitating thrombocytopenia such as those mentioned cannot be excluded. Finally, in cannot be excluded that the administration of unmatched platelet concentrates in patient 1 (given three times within a short time frame after her first drop of platelet count) contributed to the immune pathogenesis and persistence of thrombocytopenia.

There is no evidence that complementation group or position or type of mutations in the present patients predisposed to ITP. Exome sequencing in a larger series of FA patients with ITP might unveil second gene variants as a basis for the association of these apparently coincidental clinical conditions.

\section{Conclusion}

Thrombocytopenia is a common event during the natural course of FA. Because it is usually ascribed to bone marrow failure in FA, occurrence of ITP in those patients might be missed or misinterpreted. Our data show that acute and chronic ITP may occur in FA patients, and regular ITP-directed (IVIG) or "FA-adapted, ITP-directed" treatment options (such as danazol) proved successful. In contrast to current guidelines for the treatment of ITP (4), we chose the synthetic androgen danazol for the long-term treatment of chronically persisting ITP in a 2-year-old girl with FA and observed good response with tolerable side effects. With 15 months follow-up, we did not identify ITP as precursor of MDS in two pediatric FA patients.

\section{Author Contributions}

MS, MB, RD, and CU designed and coordinated the study; DS performed genetic analyses; $\mathrm{AK}$ and MS wrote the final draft of the paper; SL and MS analyzed data and prepared tables and figures; SL, MS, AW, UB, SB, CU, and MB took care of the patients; all authors read and agreed to the final version of the manuscript.

\section{Acknowledgments}

The authors thank the patient families for sharing their clinical and laboratory data and for giving their informed consent for this publication. Furthermore, attendants of the 2014 Düsseldorf Symposium on Fanconi Anemia including Prof. C. Bonfim, Prof. C. Kratz and Prof. W. Ebell are thanked for their valuable discussion of the patients' clinical courses. The study was supported by a grant of the "Steirische Kinderkrebshilfe" to MGS (A27213004019).

2. Moldovan GL, D'Andrea AD. How the Fanconi anemia pathway guards the genome. Annu Rev Genet (2009) 43:223-49. doi:10.1146/ annurev-genet-102108-134222

3. Neveling K, Endt D, Hoehn H, Schindler D. Genotype-phenotype correlations in Fanconi anemia. Mutat Res (2009) 668(1-2):73-91. doi:10.1016/j.mrfmmm. 2009.05.006 
4. Neunert C, Lim W, Crowther M, Cohen A, Solberg L Jr, Crowther MA, et al. The American Society of Hematology 2011 evidence-based practice guideline for immune thrombocytopenia. Blood (2011) 117(16):4190-207. doi:10.1182/ blood-2010-08-302984

5. Seidel MG. Autoimmune and other cytopenias in primary immunodeficiencies: pathomechanisms, novel differential diagnoses, and treatment. Blood (2014) 124(15):2337-44. doi:10.1182/blood-2014-06-583260

6. Kalb R, Neveling K, Hoehn H, Schneider H, Linka Y, Batish SD, et al. Hypomorphic mutations in the gene encoding a key Fanconi anemia protein, FANCD2, sustain a significant group of FA-D2 patients with severe phenotype. Am J Hum Genet (2007) 80(5):895-910. doi:10.1086/517616

7. Fogarty PF, Segal JB. The epidemiology of immune thrombocytopenic purpura. Curr Opin Hematol (2007) 14(5):515-9. doi:10.1097/MOH.0b013e3282ab98c7

8. Shimamura A, Alter BP. Pathophysiology and management of inherited bone marrow failure syndromes. Blood Rev (2010) 24(3):101-22. doi:10.1016/j.blre. 2010.03.002

9. Cines DB, Bussel JB, Liebman HA, Luning Prak ET. The ITP syndrome: pathogenic and clinical diversity. Blood (2009) 113(26):6511-21. doi:10.1182/ blood-2009-01-129155

10. Psaila B, Bussel JB. Refractory immune thrombocytopenic purpura: current strategies for investigation and management. Br J Haematol (2008) 143(1):16-26. doi:10.1111/j.1365-2141.2008.07275.x

11. Shimomura M, Hasegawa S, Seki Y, Fukano R, Hotta N, Ichiyama T. Intravenous immunoglobulin does not increase FcgammaRIIB expression levels on monocytes in children with immune thrombocytopenia. Clin Exp Immunol (2012) 169(1):33-7. doi:10.1111/j.1365-2249.2012.04591.x

12. Meyer O, Winter O, Salama A. Influence of intravenous immunoglobulin treatment on thrombopoiesis. Transfus Med Hemother (2012) 39(3):217-20. doi: $10.1159 / 000339258$

13. Rose SR, Kim MO, Korbee L, Wilson KA, Ris MD, Eyal O, et al. Oxandrolone for the treatment of bone marrow failure in Fanconi anemia. Pediatr Blood Cancer (2014) 61(1):11-9. doi:10.1002/pbc.24617

14. Scheckenbach K, Morgan M, Filger-Brillinger J, Sandmann M, Strimling B, Scheurlen W, et al. Treatment of the bone marrow failure in Fanconi anemia patients with danazol. Blood Cells Mol Dis (2012) 48(2):128-31. doi:10.1016/j. bcmd.2011.11.006

15. Ahn YS. Efficacy of danazol in hematologic disorders. Acta Haematol (1990) 84(3):122-9. doi:10.1159/000205048

16. Schreiber AD, Chien P, Tomaski A, Cines DB. Effect of danazol in immune thrombocytopenic purpura. N Engl J Med (1987) 316(9):503-8. doi:10.1056/ NEJM198702263160903
17. Wang WC. Evans syndrome in childhood: pathophysiology, clinical course, and treatment. Am J Pediatr Hematol Oncol (1988) 10(4):330-8. doi:10.1097/ 00043426-198824000-00013

18. Norton A, Roberts I. Management of Evans syndrome. Br J Haematol (2006) 132(2):125-37. doi:10.1111/j.1365-2141.2005.05809.x

19. Niemeyer CM, Baumann I. Classification of childhood aplastic anemia and myelodysplastic syndrome. Hematology Am Soc Hematol Educ Program (2011) 2011:84-9. doi:10.1182/asheducation-2011.1.84

20. Gillio AP, Verlander PC, Batish SD, Giampietro PF, Auerbach AD. Phenotypic consequences of mutations in the Fanconi anemia FAC gene: an International Fanconi Anemia Registry study. Blood (1997) 90(1):105-10.

21. Verlander PC, Kaporis A, Liu Q, Zhang Q, Seligsohn U, Auerbach AD. Carrier frequency of the IVS $4+4 \mathrm{~A}->$ T mutation of the Fanconi anemia gene FAC in the Ashkenazi Jewish population. Blood (1995) 86(11):4034-8.

22. Wagner JE, Tolar J, Levran O, Scholl T, Deffenbaugh A, Satagopan J, et al. Germline mutations in BRCA2: shared genetic susceptibility to breast cancer, early onset leukemia, and Fanconi anemia. Blood (2004) 103(8):3226-9. doi:10. 1182/blood-2003-09-3138

23. Alter BP, Rosenberg PS, Brody LC. Clinical and molecular features associated with biallelic mutations in FANCD1/BRCA2. J Med Genet (2007) 44(1):1-9. doi:10.1136/jmg.2006.043257

24. Myers K, Davies SM, Harris RE, Spunt SL, Smolarek T, Zimmerman S, et al. The clinical phenotype of children with Fanconi anemia caused by biallelic FANCD1/BRCA2 mutations. Pediatr Blood Cancer (2012) 58(3):462-5. doi:10. $1002 /$ pbc. 23168

25. Islam A, Rafiq S, Kirwan M, Walne A, Cavenagh J, Vulliamy T, et al. Haematological recovery in dyskeratosis congenita patients treated with danazol. $\mathrm{Br} \mathrm{J}$ Haematol (2013) 162(6):854-6. doi:10.1111/bjh.12432

Conflict of Interest Statement: The authors declare that the research was conducted in the absence of any commercial or financial relationships that could be construed as a potential conflict of interest.

Copyright (c) 2015 Karastaneva, Lanz, Wawer, Behrends, Schindler, Dietrich, Burdach, Urban, Benesch and Seidel. This is an open-access article distributed under the terms of the Creative Commons Attribution License (CC BY). The use, distribution or reproduction in other forums is permitted, provided the original author(s) or licensor are credited and that the original publication in this journal is cited, in accordance with accepted academic practice. No use, distribution or reproduction is permitted which does not comply with these terms. 